\title{
Provider Stigma Associated with Treating Drug Addiction
}

\author{
Bobbie Taylor* \\ School of Nursing, Marshall University, USA
}

*Corresponding author: Bobbie J Taylor, School of Nursing, One John Marshall Drive, Huntington, West Virginia, USA.

Received Date: July 23, 2019

Published Date: July 29, 2019

\begin{abstract}
Drug addiction has reached epidemic numbers in the United States. As the number of individuals with substance use disorders has grown, there remains a large portion of this population who do not seek treatment for their condition. Although many factors play into an individual's choice to receive treatment, one must question if provider stigma may hold a role in the limited number of individuals seeking help. An increase in knowledge and easily accessible training may help close this gap in healthcare..
\end{abstract}

Keywords: Drug addiction; Provider stigma; Medication-assisted treatment (MAT); Substance Abuse and Mental Health Services Administration (SAMHSA)

Abbreviations: SUD: Substance use disorder; MAT: Medication-Assisted Treatment; PCSS: Provider Clinical Support System; SAMHSA: Substance Abuse and Mental Health Services Administration

\section{Introduction}

In 2017, nearly 20 million American adults struggled with substance use disorder (SUD) [1]. During this year there were more than 70,200 deaths reported from overdoses, with over 17,000 from prescription opioids [2]. Even with such staggering statistics, of those 20 million individuals only 4 million people received treatment [2]. Drug addiction is considered a treatable disease [2]. It is a chronic condition, similar to hypertension (high blood pressure) and chronic obstructive pulmonary disease (COPD). Drug dependence is a complex disorder which requires specialized training to identify and treat. Research has shown recovery from drug addiction begins with treatment with medications such as Buprenorphine (Suboxone) with an additional component of behavioral therapy or counseling. As with any chronic condition, if an individual chose to stop taking their medication, then there is a risk of relapsing. Although it must be an individual's choice to receive treatment, one must wonder if there are other reasons which limit addiction treatment. One possible problem with the drug crises could lie in the stigma many healthcare providers hold regarding treatment with Buprenorphine.

\section{Discussion}

Stigma is defined as a mark of disgrace associated with a particular circumstance, quality, or person. According to the
Hazelden Betty Ford Foundation, addiction stigma prevents many individuals from receiving the help they need. Often drug addiction is not viewed as a chronic disease, but instead a moral or criminal issue. The World Health Organization (WHO) state that stigma is a major cause of "discrimination" and are based on assumptions instead of facts [3]. Although substance use disorder is an actual diagnosis, it remains "marginalized" by many in the healthcare field [4]. These patients, when compared to other patients with chronic illnesses, are often blamed for their behavior [5]. Often treatment with Buprenorphine, also referred to as Medication-Assisted Treatment (MAT), is avoided by healthcare providers because they feel these patients are "trading one addiction for another" [6]. Although research has shown MAT is effective in treating patients with substance use disorders, providers are not receptive of the facts which show these patients have a genetic predisposition and environmental causes that results in changes in the brain [7]. These changes lead to "tolerance, cravings, compulsive and destructive behaviors" [7].

Madden (2019) reports much of the stigma toward MAT is often due to limited scientific knowledge of the workings of MAT and the "evidence of its efficacy." She also feels the lack of understanding may be further promoted by inaccuracies found on the internet 
and shared through social media [6]. Other causes of stigma include inadequate training programs for nurse practitioners, physician assistants and medical doctors. It was also noted MAT providers are looked down on by providers who practice abstinent treatment options. Until providers are fully educated that addiction is a chronic disease and can be successfully treated, a gap in care for these patients will continue to exist. The American Society of Addiction Medicine has created a Provider Clinical Support System (PCSS) which is a program funded by the Substance Abuse and Mental Health Services Administration (SAMSHA) to provide treatment and mentoring to healthcare providers in an effort to combat the opioid epidemic. This PCSS site offers educational webinars, coaching, and resources to educate others in regard to treatment of substance use disorders.

Another barrier preventing providers from treating patients with substance use disorder is the fear of having "those people" in the waiting room. Unfortunately, "those people" are already in the waiting room. Addiction does not discriminate. We see doctors, nurses, laborers, accountants and retired individuals who are addicted to opioids. These patients are being seen for other chronic illnesses such as hypertension and diabetes. If providers could receive evidence-based training on the prescribing and effectiveness of MAT, their patients could receive a one-stop healthcare service. This might lead to more patients seeking treatment for substance use disorder.

\section{Conclusion}

In conclusion, the offer of free continuation education credits and simplifying the access to training seminars may be beginning steps to help lessen the stigma of MAT. As one reminisces to a simpler time, Saturday morning cartoons may come to mind. The educational short, School House Rock would end with the statement, "Knowledge is Power." Knowledge is power. If we as healthcare providers would take a vested interest in educating ourselves and our fellow providers, we may be able to reduce the stigma associated with drug addiction treatment and gain control of the drug epidemic.

\section{Acknowledgment}

None.

\section{Conflict of Interest}

Author declare no conflicts of interest to report.

\section{References}

1. Scot Thomas (2019) Alcohol and Drug Abuse Statistics. American Addiction Centers.

2. (2019) Overdose Death Rates. National Institute on Drug Abuse: Advancing Addiction Science.

3. Substance Abuse. World Health Organization (WHO).

4. Smashing the stigma of addiction. Hazelton Betty Ford Foundation.

5. Livingston JD, Milne T, Fang ML, Amari E (2012) The effectiveness of interventions for reducing stigma related to substance use disorders: a systematic review. Addiction 107(1): 39-50.

6. Madden E (2019) Intervention stigma: How medication-assisted treatment marginalizes patients and providers. Social Science \& Medicine 232(2019): 324-331.

7. Providers Clinical Support System. American Society of Addiction Medicine. 